\title{
INFRARED ABSORPTION STUDY OF THERMALLY GENERATED SHALLOW DONOR CENTERS IN CZOCHRALSKI SILICON
}

\author{
P. Kaczor, K. Kopalko, M. Godlewski
}

Institute of Physics, Polish Academy of Sciences

Al. Lotników 32/46, 02-668 Warszawa, Poland

AND T. GregorkiEWICZ

Van der Waals-Zeeman Laboratorium, University of Amsterdam

Valckenierstraat 65, 1018 XE Amsterdam, The Netherlands

A comparative study of thermally generated donor centers in boron and aluminum doped Czochralski silicon was performed by means of Fourier transform infrared technique. A detailed study revealed presence of donor centers belonging to the well-known series of thermal donors and shallow thermal donors. For both types of material the same centers could be observed while considerable differences in their generation kinetics occurred. In addition to the previously identified species also new ones could be observed. One of them, with single ionization level at approximately $39.5 \mathrm{meV}$, was found to exhibit clear dependence of its concentration upon illumination of the sample during cooling from room temperature to liquid He temperature.

PACS numbers: $71.55 . \mathrm{Ht}, 78.50 . \mathrm{Ge}$

\section{Introduction}

It is a well-known phenomenon that shallow donor centers are produced upon exposure of oxygen-rich silicon to $350-500^{\circ} \mathrm{C}$ heat treatment. These are termed thermal donors TD's and have been extensively studied over the last three decades [1]. Unfortunately, the phenomenon of thermal donors appears to be very complex and consequently no undisputed microscopic model of the TD center exists at the moment [2]. Nevertheless, an immense amount of information regarding the thermal donor issue has been gathered. Here a very important contribution comes from the infrared absorption studies. The absorption studies [3] revealed the two most important features of TD: its effective mass theory (EMT), shallow double donor character and the existence of several, very similar species. This information was later somewhat complicated by photoionization spectroscopy findings [4] which revealed that in high-resistivity material another series of (single) donors, termed 
shallow thermal donors STD's, could be created by heat treatment identical to that generating TD series. This particular report is still a reason of considerable confusion as the (microscopic) origin of STD centers is being searched for.

In the past magnetic resonance studies helped to establish structural and electronic models of numerous defect centers. Therefore, also EPR and ENDOR studies for TD centers have been performed [5-7]. Unfortunately, most probably due to a very delocalized character of these centers, magnetic studies did not provide conclusive answers. At the same time they have risen serious doubts as to whether aluminum doping did not lead to yet one more variation of TD center, namely in the form of aluminum-oxygen agregate. In the current study this particular issue is investigated by comparing the thermal donor centers as generated in boron and aluminum doped silicon.

\section{Experimental results and discussion}

Following the aim of the project two kinds of starting material were used. The materials labeled W38 and W39 were high quality commercial Czochralski-grown low-carbon silicon (WASO grade) doped with aluminum $\left(4 \times 10^{15} \mathrm{~cm}^{-3}\right)$ and boron $\left(1.3 \times 10^{16} \mathrm{~cm}^{-3}\right)$, respectively. After the initial oxygen dispersion heat treatment the samples were subsequently annealed at $470^{\circ} \mathrm{C}$ for various time intervals. The infrared absorption measurements were performed at liquid helium temperature with the Bomem FTIR spectrometer.

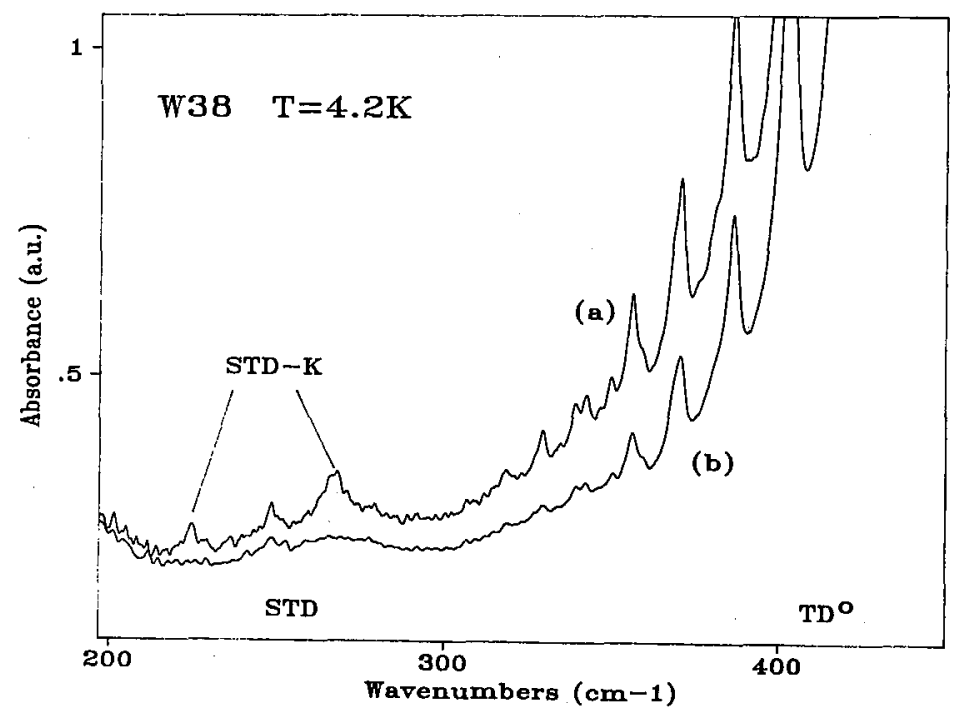

Fig. 1. Absorption spectra of the W38 sample annealed for 5.5 hours. (a) Spectrum measured after cooling under illumination. (b) Spectrum measured after cooling in dark.

The main conclusion which can be drawn from the present study is that regardless of the actual dopant present in the sample the same variety of TD 
Identification of spectral lines of TD and STD.

TABLE

\begin{tabular}{c|l|l|l|l|c|c}
\hline \hline \multicolumn{5}{c|}{ Transition } & \multicolumn{2}{c}{$\nu_{1 s-2 p_{+}-\nu_{1 s-2 p_{0}}}$} \\
(expcrimcnt)
\end{tabular}

and STD centers is being generated. Indeed, while considerable differences appear to be in the generation kinetics of individual centers, the centers themselves are clearly the same and can be identified with these reported in the earlier studies $[3,4]$. Table lists the positions of various transitions of TD and STD species as observed in both kinds of materials leaving no doubt that the same centers are observed in both cases. In addition to the known centers Table lists also several new transitions. For the TD series these correspond to three still shallower species, labeled here TD-J, K, and L, whose existence has indeed been postulated before [8].

For the STD series, which appears much stronger in aluminum than in boron doped material, the present study reveals two prominent absorption lines which can be identified with an EMT-like, single donor of $\approx 39.5 \mathrm{meV}$ ionization energy. To the best of our knowledge, this center, labeled here STD-K, is reported here for the first time. It was further found that the observation of the STD-K center depends upon the cooling procedure of the sample. Figure 1 presents comparison of two absorption spectra of the same sample cooled under different conditions: in the 
dark and upon illumination. As can be concluded, the STD-K center appears only for the illuminated sample. Such behavior is somewhat similar to the bistability as observed before [3] for the two deepest TD species, but clearly requires further studies before its origin is firmly established. It is also only fair to note here that the observation of this new STD-K species does not really fit into the accepted STD generation scheme. According to it, in contrast to TD's, the deeper species are generated subsequently upon prolonged annealing while the STD-K donor with its largest ionization energy can be observed already after (very) short annealing times.

The work currently in progress, and then especially the correlation of the infrared absorption data with the results obtained by photoluminescence, will further address the issues discussed in this paper with special emphasis on the mutual relation between TD and STD series.

\section{References}

[1] L.C. Kimerling, in: Proc. Symp. Oxygen, Carbon, Hydrogen Nitrogen Cryst. Silicon, Vol. 59 of Materials Research Society Symposia Proceedings, Eds. J.C. Mikkelsen Jr., S.J. Pearton, J.W. Corbett, S.J. Pennycook, MRS, Pittsburgh 1986, p. 83.

[2] P. Deák, M. Heinrich, L.C. Snyder, J.W. Corbett, Mater. Sci. Forum 83-87, 395 (1992).

[3] P. Wagner, J. Hage, Appl. Phys. A 49, 123 (1989).

[4] J.A. Griffin, H. Navarro, J. Weber, L. Genzel, J.T. Borenstein, J.W. Corbett, L.C. Snyder, J. Phys. C 19, L579 (1986).

[5] T. Gregorkiewicz, H.H.P.Th. Bekman, C.A.J. Ammerlaan, Phys. Rev. B 38, 3998 (1988).

[6] H.H.P.Th. Bekman, T. Gregorkiewicz, C.A.J. Ammerlaan, Phys. Rev. B 39, 1648 (1989).

[7] J. Michel, J.R. Niklas, J.-M. Spaeth, Phys. Rev. B 40, 1732 (1989).

[8] G. Pensl, private communication. 